\title{
Upregulation of miR-146a by YY1 depletion correlates with delayed progression of prostate cancer
}

\author{
YEQING HUANG $^{1 *}$, TAO TAO $^{2 *}$, CHUNHUI LIU $^{1 *}$, HAN GUAN $^{1 *}$, GUANGYUAN ZHANG $^{1}$, \\ ZHIXIN LING ${ }^{1}$, LEI ZHANG ${ }^{1}$, KAI LU ${ }^{1}$, SHUQIU CHEN ${ }^{1}$, BIN XU ${ }^{1}$ and MING CHEN ${ }^{1}$ \\ ${ }^{1}$ Department of Urology, Affiliated Zhongda Hospital, Medical School of Southeast University, Nanjing, Jiangsu; \\ ${ }^{2}$ Department of Urology, Anhui Provincial Hospital, Anhui Medical University, Hefei, Anhui, P.R. China
}

Received October 16, 2016; Accepted December 28, 2016

DOI: $10.3892 /$ ijo.2017.3840

\begin{abstract}
Previously published studies explained that the excessive expression of miR-146a influences the prostate cancer (PCa) cells in terms of apoptosis, progression, and viability. Although miR-146a acts as a tumor suppressor, current knowledge on the molecular mechanisms that controls its expression in PCa is limited. In this study, gene set enrichment analysis (GSEA) showed negatively enriched expression of miR-146a target gene sets and positively enriched expression of gene sets suppressed by the enhancer of zeste homolog 2 (EZH2) after YY1 depletion in PCa cells. The current results demonstrated that the miR-146a levels in PCa tissues with high Gleason scores $(>7)$ are significantly lower than those in PCa tissues with low Gleason scores $(\leq 7)$, which were initially observed in the clinical specimens. An inverse relationship between YY1 and miR-146a expression was also observed. Experiments indicated the decrease in cell viability, proliferation, and promoting apoptosis after YY1 depletion, while through inhibiting miR-146a could alleviate the negative effect brought by YY1 depletion. We detected the reversed adjustment of YY1 to accommodate miR-146a transcriptions. On the basis of YY1 depletion, we determined that the expression of miR-146a increased after EZH2 knockdown. We validated the combination of YY1 and its interaction with EZH2 at the miR-146a promoter binding site, thereby prohibiting the transcriptional activity of miR-146a in PCa cells. Our results suggested that YY1 depletion repressed PCa cell viability and proliferation and induced apoptosis at least in a miR-146a-assisted manner.
\end{abstract}

Correspondence to: Professor Bin Xu or Professor Ming Chen, Department of Urology, Affiliated Zhongda Hospital, Medical School of Southeast University, 87 Dingjia Bridge Road, Nanjing, Jiangsu 210009, P.R. China

E-mail: xb15896450810@126.com

E-mail: mingchenseu@126.com

*Contributed equally

Key words: prostate cancer, miR-146a, YY1, enhancer of zeste homolog 2

\section{Introduction}

Prostate cancer ( $\mathrm{PCa}$ ) has been reported globally as a type of cancer exclusively diagnosed in males and is defined as one of the most common malignant tumors in European and American countries (1). PCa easily and biochemically relapses along with the development of hormone refractory cancer, and such condition remains to be a major cause of poor prognosis (2).

MicroRNAs (miR), as a key regulator, have been implicated as regulators controlling diverse biological processes at the post-transcriptional level of repression $(3,4)$. Previously published studies explained that the excessive expression of miR-146a influenced the PCa cells in terms of apoptosis, progression, and viability by directly targeting the epidermal growth factor receptor (EGFR) (5), Ras-related C3 botulinum toxin substrate 1 (Rac1) (6), ROCK1 (7), interleukin-1 receptor-associated kinase 1 (IRAK1), and tumor necrosis factor receptor-associated factor 6 (TRAF6) (8). Although miR-146a acts as a tumor suppressor in PCa cells, current knowledge on the molecular mechanisms that contribute to the expression of miR-146a in PCa cells is still limited. The probable molecular mechanisms that contribute to the aberrant expression of miRNAs result from gene mutation, epigenetic modification, and alteration of Dicer abundance. By means of promoter analysis, miR-146a was predicted to be transcriptionally regulated by the vital transcriptional factor YY1. YY1, a uniquely expressed zinc finger transcriptional factor, played a double-trend regulatory role in a variety of biological processes, such as embryonic development, cell growth, apoptosis, differentiation, and oncogenic transformation (9-11).

In this study, we determined that the decreased PCa cell viability, proliferation and increased apoptosis caused by YY1 depletion accompanied by upregulation of miR-146a. We further validated the combination of YY1 and its interaction with EZH2 at the miR-146a promoter prohibited the transcriptional activity of miR-146a in PCa cells. Together with preceding results concluded by our team $(5-7,12)$, our findings provided an in-depth understanding of the correlation of YY1/EZH2 and miR-146a in PCa cell function disorders, thereby potentially becoming a therapeutic target for PCa treatment. 


\section{Materials and methods}

Cell culture. The cell lines were obtained from the Shanghai Academy of Life Sciences, which is affiliated with the Chinese Academy of Sciences. PC3 and DU145 human PCa cells were cultured with DMEM/F12 (1:1) medium supplied with $10 \%$ fetal bovine serum and antibiotics at $37^{\circ} \mathrm{C}$ in a $5 \%$ $\mathrm{CO}_{2}$ incubator.

Tissue samples. A total of 34 tissue samples were obtained between 2012 and 2015 from the radical prostate resection of 34 patients with PCa admitted to the Zhongda Hospital affiliated with the Southeast University. The patients were informed about the study and were asked to sign consents for their tissues used in the scientific research. Ethical approval for the current study was previously obtained from the Zhongda Hospital. The diagnoses were based on the pathological evidence, and the histological features of the specimens were evaluated by the senior pathologist according to the classification criteria of the World Health Organization.

Animal experiments. BALB/c nude male mice (six-week-old) were purchased from Shanghai SLAC Laboratory Animal Company. Animal experiments were conducted in accordance with the protocol approved by the National Institutes of Health Guide for the Care and Use of Laboratory Animals. PC3 cells were transfected with sh-GFP-YY1 or sh-GFP-NC lentivirus to establish a stabilized cell line. A $100 \mu$ l cell culture mixed with $10^{6}$ cells was subcutaneously injected into each flank of the mice. Thereafter, tumor size was measured every week. All mice were sacrificed on the 29th day. The tumors were removed fresh in preparation for miR-146a detection.

ONCOMINE database reanalysis. The original YY1 mRNA expression data and the prognostic data of YY1 in PCa specimens were retrieved from the ONCOMINE database (www.oncomine.org) and GEO database (Nakagawa prostate, GSE10645, https://www.ncbi.nlm.nih.gov/geo/query/acc. cgi?acc=GSE10645. Grasso prostate, GSE35988, http://www. ncbi.nlm.nih.gov/geo/query/acc.cgi?acc=GSE35988. Lapointe prostate, GSE3933, https://www.ncbi.nlm.nih.gov/geo/query/ acc.cgi?acc=GSE3933.). Re-analyses included the relationship among YY1 expression level, biochemical recurrence within five years, and Gleason scores. These processes were performed to determine the potential relationship between PCa prognosis and YY1 expression levels.

$R N A$ isolation and quantitative real-time polymerase chain reaction ( $q R T-P C R$ ). Total RNA was extracted from tissues or cells (after transfection for $72 \mathrm{~h}$ ) using TRIzol (Takara, Shiga, Japan) according to the manufacturer's instruction. RNA concentration was measured by NanoDrop2000 (Thermo Fisher Scientific, Waltham, MA, USA). For miR-146a, total RNA (10 ng) was reversed into cDNAs. The qRT-PCR for miR-146a was performed using the SYBR Green PCR Master Mix of the Hairpin-it miRNAs RT-PCR Quantitation kit (GenePharma, Shanghai, China) according to the manufacturer's protocols. The following PCR conditions were used for detecting miR-146a: $95^{\circ} \mathrm{C}$ for $3 \mathrm{~min}, 40$ cycles at $95^{\circ} \mathrm{C}$ for $12 \mathrm{sec}$, and $62^{\circ} \mathrm{C}$ for $40 \mathrm{sec}$ (running in the ABI Step PCR
System). The expression of U6 was used as internal control. The relative expression of miR-146a compared with U6 was calculated using the $2^{-\Delta \Delta C t}$ method.

RNA sequencing and bioinformatics analysis. We depleted YY1 to establish a stabilized sh-YY1-PC3 cell line (targeting sequences are listed in Table I) to assess the role of YY1 in PCa cells. Stable cell lines were established by puro-mycin. RNA sequencing was assigned to and performed by Huada Genomics Technology Co. (Shenzhen, China). RNA samples extracted and assigned to RNA-sequencing using Illumina-HiSeq2000 system is $500 \mu \mathrm{g}$ for sh-YY1 and sh-NC respectively. GSEA was used to identify the pathway or gene sets that are correlated with the YY1 expression profile (http://www.broadinstitute.org/ gsea/index.jsp). The gene sets were acquired from the Molecular Signatures Database on that website. The normalized enrichment score (NES) and false discovery rate (FDR) were used to compare the results of the analysis across gene sets.

YY1 overexpression and RNA silencing. Human YY1 overexpression pCMV6-YY1 vectors were obtained from Sangon Biotech Co., Ltd., Shanghai, China. The human siRNA-YY1 sequence was purchased from GenePharma. Table I lists the detailed sequences of siRNAs and miR-146a. The transfection process was performed as previously described (5).

MTT assay. The transfected cells were plated at 2,500 cells/well in 96-well plates. A total of $20 \mu \mathrm{l} \mathrm{of} 5 \mathrm{mg} / \mathrm{ml}$ MTT in PBS was added to each well every $24 \mathrm{~h}$ after plating. The cells were lysed after $2 \mathrm{~h}$ with the addition of $200 \mu \mathrm{l}$ dimethyl sulfoxide (Sigma-Aldrich, St. Louis, MO, USA). Absorbance was measured at $570 \mathrm{~nm}$.

Colony formation assay. After transfection, the cells were seeded in six-well plates at a density of 800 cells/well and cultured for 10-14 days until colonies appeared. Thereafter, the cells were stained with crystal violet. The number of colonies was counted only when they contained more than 50 cells.

Cell apoptosis. Cell apoptosis was detected by a fluorescenceactivated cell sorter. After $72 \mathrm{~h}$ of transient transfections, the cells were harvested by trypsinization, and cell apoptosis was induced and measured as previously described (7).

In situ hybridization (ISH) and immunohistochemical staining (IHC). ISH and IHC were performed, and subsequent results were evaluated as previously described (13). ISH was performed using antisense locked nucleic acid (LNA)-modified probe based on the sequence of hsa-miR146a (Boster, Wuhan, China). Table I lists the detailed LNA sequence. IHC was performed using YY1 antibody (1:250; Proteintech, Chicago, IL, USA) according to the manufacturer's instructions. Original magnification, x200. Evaluation of ISH and IHC was as follows: Sections with unlabeled or labeled cells $<5 \%$ were scored 0 . Sections with $5-30 \%$ of labeled cells were scored 1, those with $31-70 \%$ of labeled cells were scored 2 , and those with $\geq 71 \%$ of labeled cells were scored 3 . The staining intensity was scored similarly using 0 for negative staining, 1 for weakly positive, 2 for moderately positive, and 3 for strongly positive. The scores 
Table I. Sequences of si-RNAs, sh-YY1, LNA for miR-146a and primers of ChIP and Luciferase assay.

\begin{tabular}{ll}
\hline Item & \\
\hline si-YY1 & Sequence $\left(5^{\prime}-3^{\prime}\right)$ \\
si-NC & CGAGGAUCAGAUUCUCAUC \\
si-EZH2 & UUCUCCGAACGUGUCACGU \\
sh-YY1 & GAAUGGAAACAGCGAAGGATT \\
sh-NC & CGAGGATCAGATTCTCATC \\
anti-miR-146a & TTCTCCGAACGTGTCACGT \\
LNA for ISH & AACCCAUGGAAUUCAGUUCUCA \\
CHIP primer F & ACTCTTGACTTAAGGTACCCAA \\
CHIP primer R & CCTTCAGGGGAGGTCAGGAAAGTGA \\
Luciferase primer F & CTTTGGTGAGGTTAGGGGGACTTGC \\
Luciferase primer R & CGAGGTACCTGGGAGCTCAGCAAGATAAGCAAC \\
\hline
\end{tabular}

F, forward; R, reverse.

for the percentage of positive tumor cells and for the staining grade were calculated to generate an immune-reactive score for each specimen. The product of the quantity and intensity scores was calculated, such that a final score of $0-1$ indicates negative expression (-), 2-3 indicates weak expression (+), 4-5 indicates moderate expression $(++)$, and 6 indicates strong expression $(+++)$. Each sample was examined separately and scored by two researchers.

Luciferase reporter assay. Promoter sequences (14) for miR-146a was reported previously, and were retrieved from NCBI Genome Bioinformatics (http://www.ncbi.nlm.nih. gov/). Prediction of the transcription factor binding sites was performed using the JASPAR analysis tool (http://jaspar. genereg.net/). The promoter segment of hsa-miR-146a was amplified by PCR $\left(98^{\circ} \mathrm{C}\right.$ for $2 \mathrm{~min}, 98^{\circ} \mathrm{C}$ for $15 \mathrm{sec}, 68^{\circ} \mathrm{C}$ for $2 \mathrm{~min}$ with 35 cycles, and $68^{\circ} \mathrm{C}$ for $5 \mathrm{~min}$ ) using human genome DNA. The PCR products were cloned into the KpnI and HindIII restriction sites (primer sequences are presented in Table I), downstream of the open reading frame of luciferase in the pGL3-basic-Vector (Promega, Madison, WI, USA), to generate the pGL3-basic-miR-146a promoter reporter. For reporter assays, cells were transfected with pGL3-miR-146a promoter or mutant reporter plasmid and pRL-TK plasmid. Luciferase assay activity was measured $48 \mathrm{~h}$ after transfection using the Dual-Luciferase Reporter Assay System (Promega).

Chromatin immunoprecipitation (ChIP). Samples were obtained from PC3 cells (after transfection with si-NC or si-YY1 for $72 \mathrm{~h}$ ) and processed according to the manufacturer's protocol (Millipore, Billerica, MA, USA). Briefly, the chromatin solution was pre-cleared with $50 \mu \mathrm{l}$ of protein A-agarose beads (Beyotime, Shanghai, China). ChIP assays were performed using YY1 antibody (1:50, Abcam, Cambridge, UK), anti-polymerase II (Beyotime) and IgG (Beyotime), and allowed to incubate overnight at $4^{\circ} \mathrm{C}$. The precipitated samples were analyzed by PCR (expected segment length, 253 bp), and the sequences of the primers used for miR-146a promoter region are listed in Table I.

Immunofluorescence (IF). PC3 cells were cultivated on coverslips, fixed with $4 \%$ formaldehyde for $20 \mathrm{~min}$, permeabilized for $10 \mathrm{~min}$ in $0.1 \%$ Triton X-100 in PBS, and incubated for $2 \mathrm{~h}$ in blocking buffer (10\% normal goat serum and $0.3 \%$ Triton X-100 in PBS) at regular temperature. Afterward, the cells were incubated with rabbit YY1 antibody (1:250) and mouse EZH2 antibody (1:250) overnight at $4^{\circ} \mathrm{C}$. The coverslips were washed and incubated with AlexaFluor 488-conjugated anti-mouse and AlexaFluor 594-conjugated anti-rabbit immunoglobulin G (Invitrogen, Waltham, MA, USA) and mounted with the mounting medium (ProLong Gold Antifade Reagent; Invitrogen). Fluorescent images were acquired using the Olympus FV 1000 microscope.

Western blot analysis and co-immunoprecipitation (Co-IP). PC3/DU145 cells were cultured and plated in six-well plates $\left(5 \times 10^{5}\right.$ cells/well). After transfection for $72 \mathrm{~h}$ with siRNA-YY1, anti-miR-146a, p-CMV6-YY1, or negative control, the cells were harvested and homogenized with sodium dodecyl sulfate (SDS) lysis buffer. The protein concentration was measured by the BCA method (Beyotime). Total protein was separated by denaturing with $10 \%$ SDS-polyacrylamide gel electrophoresis. Total cell extracts were pre-cleared with Protein $\mathrm{A}+\mathrm{G}$ beads (Beyotime) at $4^{\circ} \mathrm{C}$ for $2 \mathrm{~h}$. The supernatant was incubated with the anti-EZH2 or anti-YY1 (EZH2, 1:200; YY1, 1:200; Proteintech, Chicago, IL, USA) with gentle shacking for $10 \mathrm{~h}$ at $4^{\circ} \mathrm{C}$ followed by addition of $30 \mu \mathrm{l}$ of Protein $\mathrm{A}+\mathrm{G}$ beads for another $2 \mathrm{~h}$. The beads were re-suspended in $100 \mu \mathrm{l}$ of $2 \mathrm{X}$ loading buffer and boiled for $10 \mathrm{~min}$. Then western blot assay was performed according to the instructions previously described to detect the YY1 and EZH2. The primary antibodies (EZH2, 1:1000; YY1, 1:1000; Proteintech) and glyceraldehyde 3-phosphate dehydrogenase (GAPDH) (1:1,000, Proteintech) were purchased. 

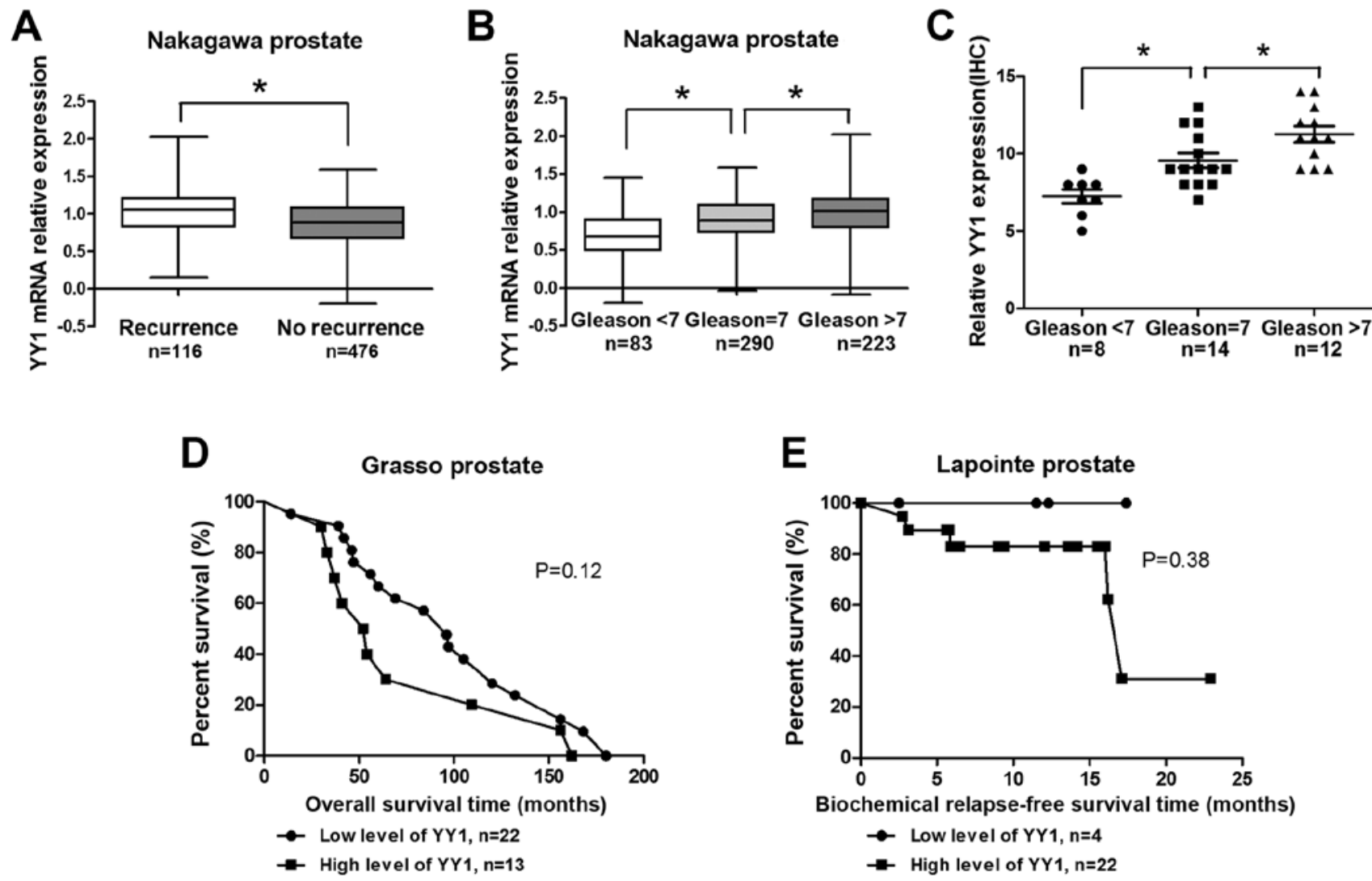

Figure 1. ONCOMINE database reanalysis and clinical significance of YY1 in PCa specimens. (A) Co-relationship between YY1 expression and biochemical recurrence by reanalysis of the Nakagawa prostate. (B) Co-relationship between YY1 expression and Gleason scores by reanalysis of the Nakagawa prostate. (C) Co-relationship between YY1 expression (IHC) and Gleason scores in $34 \mathrm{PCa}$ specimens. "P<0.05. Kaplan-Meier curves of overall survival (OS) time in Grasso prostate project (D) and biochemical relapse-free survival (BRS) time in Lapointe prostate project (E) stratified by the tissues of YY1 levels.

Statistical analysis. Each experiment was repeated at least thrice. Numerical data were presented as the mean \pm SEM. The difference among the means was analyzed using Student's t-test. One-way analysis of variance test was used to compare mean values between more than two groups. All statistical analyses were performed using SPSS 19.0 (Chicago, IL, USA). Differences were considered significant at $\mathrm{P}<0.05$.

\section{Results}

ONCOMINE database reanalysis and clinical significance of YY1 in PCa specimens. Reanalysis of the Nakagawa prostate indicated that the YY1 expression level was high in the biochemical recurrence group (Fig. 1A). In addition, the YY1 expression levels increased gradually with increasing Gleason scores (cutline=7) (Fig. 1B). In the analysis of 34 clinical PCa specimens from Zhongda Hospital, YY1 scores increased significantly with considerably high Gleason scores (cutline $=7$ ) (Fig. 1C). Results above were statistically significant. Reanalysis of correlation of YY1 and overall survival (OS) time in Grasso prostate project indicated that patients with high level of YY1 showed shorter OS time ( $\mathrm{P}=0.12$, Fig. 1D). Moreover, patients with high level of YY1 showed shorter biochemical relapse-free survival (BRS) ( $\mathrm{P}=0.38$, Fig. 1E). The results (Fig. 1D-E) were not statistically significant due to the small sample limit. However, these results suggested YY1 might play a critical role in the PCa progression.
Biological insight into the co-relationship between YYI and $m i R-146 a$ in PCa. RNA sequencing and GSEA provided information on the enriched expression of gene sets. When YY1 was knocked down in PC3 cells, the expression of miR-146a target genes demonstrated the same trend in gene enrichment, indicating the potential co-relationship between YY1 and miR-146a (NES= $-1.039, \mathrm{FDR}=0.267$ and $\mathrm{P}=0.15)$ (Fig. 2A). Then, we evaluated the miR-146a expression data through ISH and assessed the YY1 expression data through IHC of $34 \mathrm{PCa}$ specimens to investigate their co-relationship. Spearman correlation analysis showed a significantly inverse correlation between miR-146a and YY1 $(r=-0.457, \mathrm{P}=0.007)$ (Fig. 2B and C). Hence YY1 may participate in the regulation of miR-146a expression process.

Knockdown of YY1 inhibits PCa cell growth and colony forming in a miR-146a-assisted manner. YY1 was downregulated with si-YY1 to explore the effect of YY1 on PCa cell lines. In return, YY1 was upregulated when transfected with pCMV6-YY1. The knockdown or overexpression efficiency was verified through western blot analysis (Fig. 3A-C). The inhibitory efficiency for miR-146a after YY1 depletion was verified through qRT-PCR (Fig. 3D and E). The MTT and colony formation assays were used to detect the importance of miR-146a in YY1-associated PCa cell growth and proliferation. YY1 depletion significantly inhibited the growth of $\mathrm{PCa}$ cells at 48, 72, and $96 \mathrm{~h}(\mathrm{P}<0.05)$ based on the MTT assay. 
A

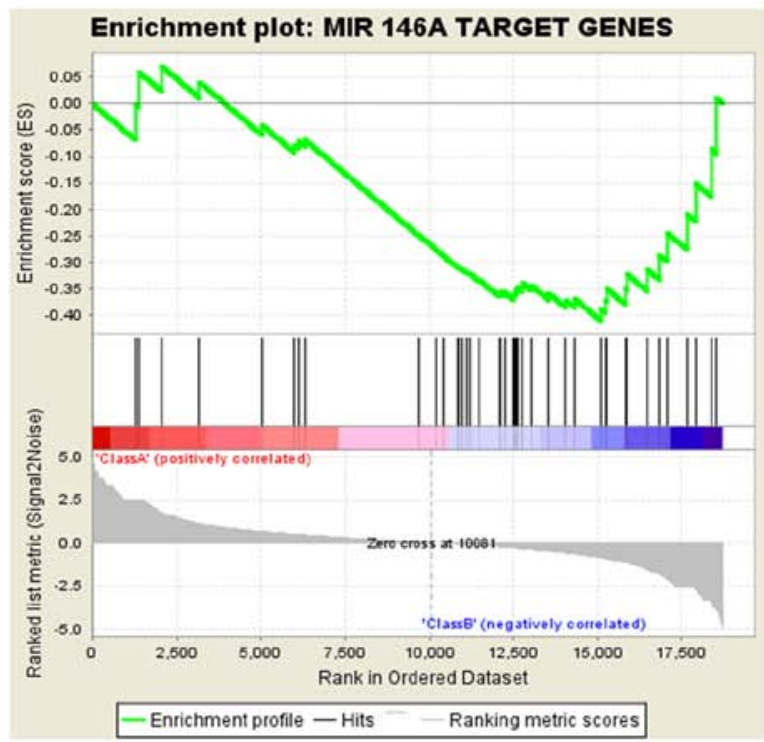

B

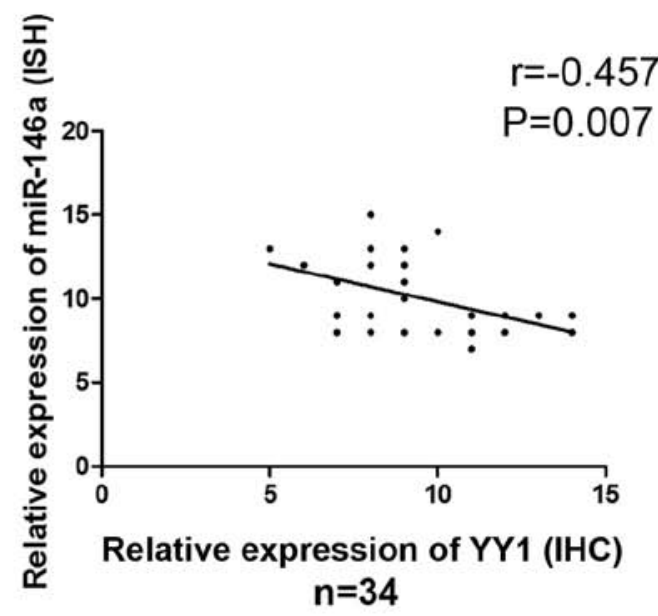

C

miR-146a (ISH)
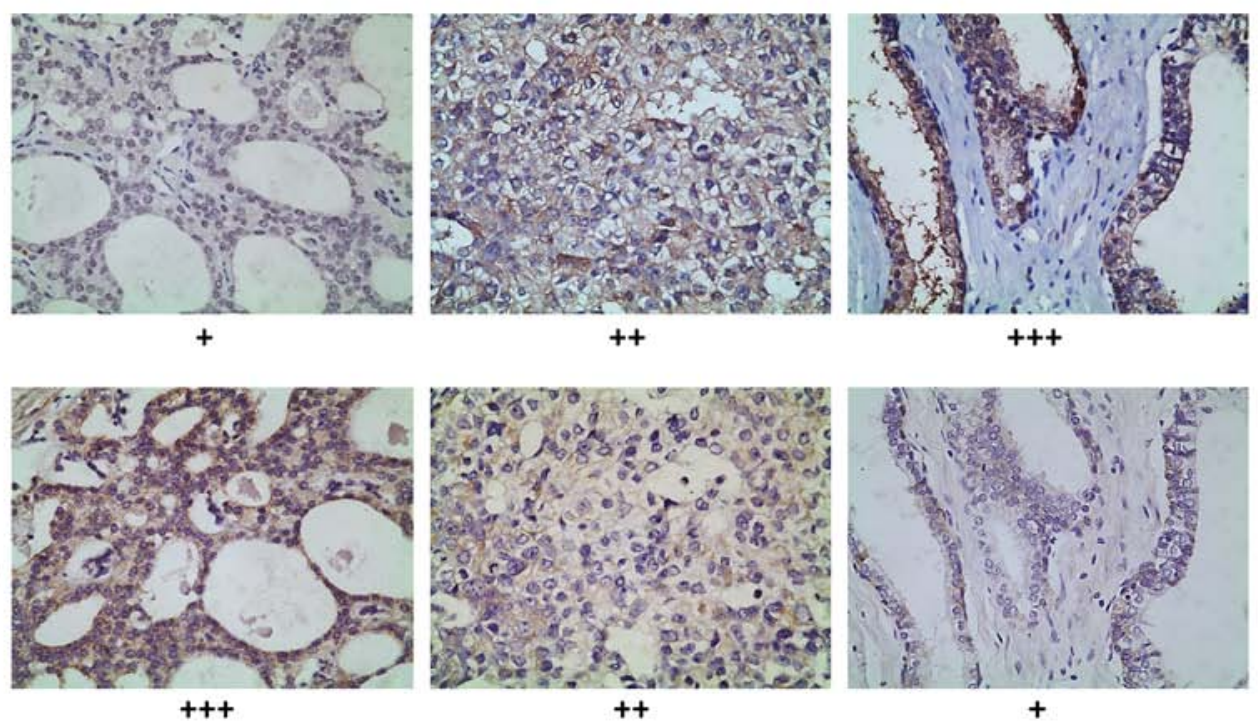

Figure 2. Biological insight into the co-relationship between YY1 and miR-146a. (A) GSEA revealed enriched expression of miR-146a target gene sets after YY1 depletion in PC3. (B) Spearman correlation analysis was performed to assess the correlation between miR-146a expression (ISH) and YY1 expression (IHC) in $34 \mathrm{PCa}$ specimens. (C) Representative images of miR-146a expression (ISH) and YY1 expression (IHC) in PCa tissues. The positive brown signals indicate the relative expression levels of YY1 and miR-146a. Original magnification, x200.

However, no significant inhibition was observed in the cells transfected with pCMV6/pCMV6-YY1 (P>0.05). Functional experiments were performed on the YY1-depleted cells supplemented with anti-miR-146a (miR-146a inhibitor) to confirm whether miR-146a was required for the YY1-mediated decrease in cell viability and proliferation. Significant results were observed when anti-miR-146a was transfected after YY1 depletion at 48, 72, and $96 \mathrm{~h}(\mathrm{P}<0.05)($ Fig. 3F-K). YY1 depletion significantly inhibited the proliferation of $\mathrm{PCa}$ cells based on colony formation assay and anti-miR-146a supplementary alleviated the inhibition effect (Fig. 3L-N). GSEA showed that a negatively enriched expression of gene sets was involved in the cell proliferation of YY1 knocked down cells $(\mathrm{NES}=-1.17$, $\mathrm{FDR}=0.22$ and $\mathrm{P}=0.11$ ) (Fig. 3O). Collectively, these results indicated that YY1 depletion inhibited cell viability and proliferation of PCa cells at least in a miR-146a-assisted manner.
Knockdown of YY1 induces cell apoptosis in miR-146a-assisted manner. YY1 was downregulated by si-YY1 and upregulated by transfection with pCMV6-YY1 to assess the role of YY1 in PCa cell apoptosis. Unobtrusive difference was observed when the cells were treated with pCMV6YY1/pCMV6 $(\mathrm{P}>0.05)$. YY1 depletion increased the apoptosis rate of the PCa cells (apoptosis rate: $5.9 \pm 0.6 \%$ vs. $13.9 \pm 0.8 \%$ in PC3 and $5.2 \pm 0.5 \%$ vs. $12.9 \pm 0.5 \%$ in DU145, $\mathrm{P}<0.05$ ), whereas supplementing the cells with miR-146a inhibitor alleviated the apoptosis effect (apoptosis rate: $14.4 \pm 1.0 \%$ vs. $9.7 \pm 0.8 \%$ in PC 3 and $13.5 \pm 0.5 \%$ vs. $10.6 \pm 0.9 \%$ in DU145, $\mathrm{P}<0.05$ ) (Fig. 4A-C). GSEA showed that a positively enriched expression of gene sets was involved in hallmarks of apoptosis $(\mathrm{NES}=1.30, \mathrm{FDR}=0.24$ and $\mathrm{P}=0.09)$ (Fig. 4D), and v-Ki-ras2 Kirsten rat sarcoma viral oncogene homolog $(\mathrm{KRAS})(\mathrm{NES}=-1.97, \mathrm{FDR}=0.04$ and $\mathrm{P}=0.01)$ 


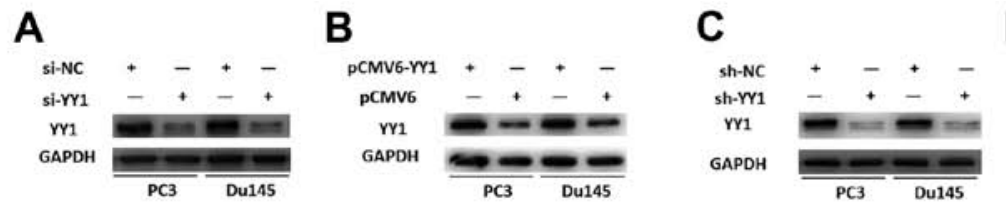

$\mathbf{F}$

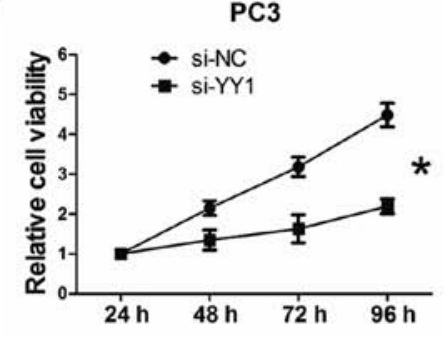

I

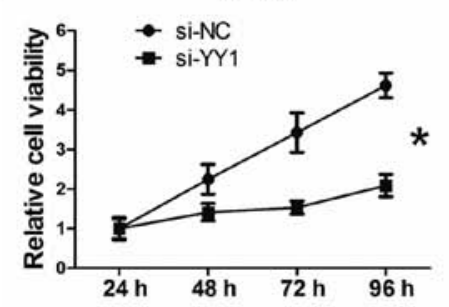

$\mathbf{L}$

PC3

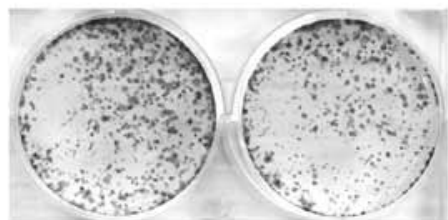

DU145

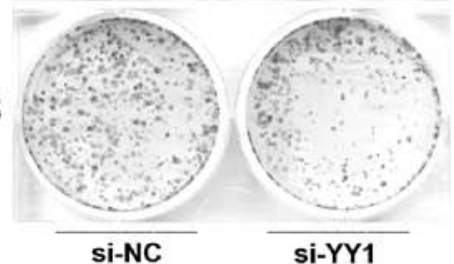

G

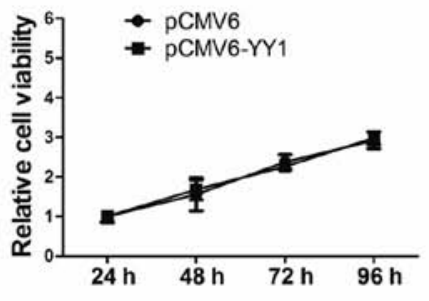

$\mathbf{J}$

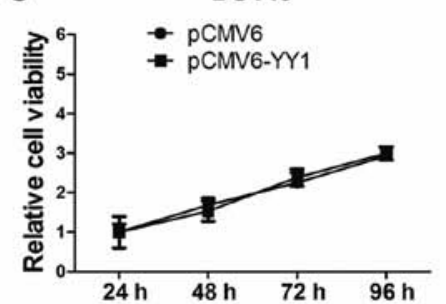

$E$ :

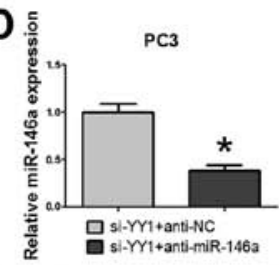

H

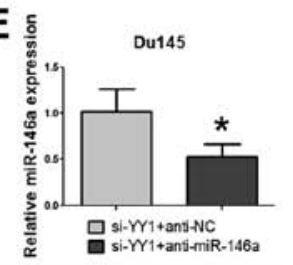

PC3

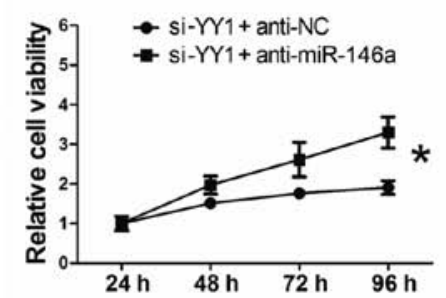

$\mathbf{K}$
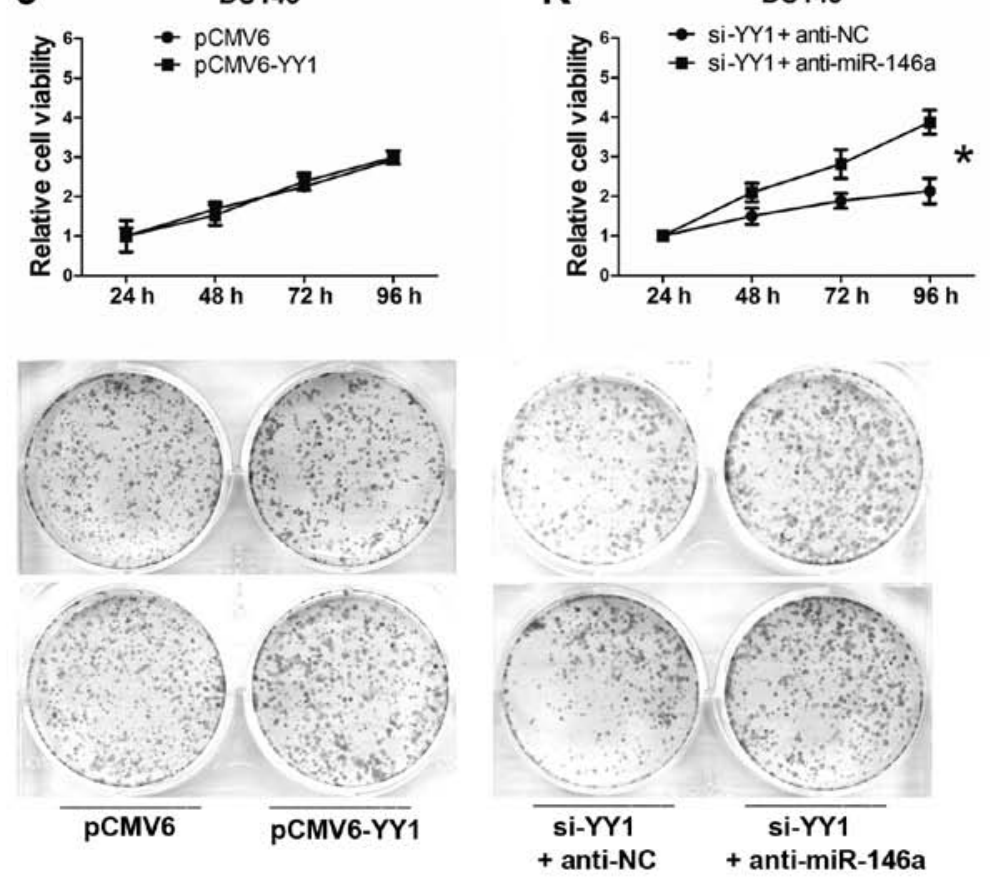

M

$\mathbf{N}$

O

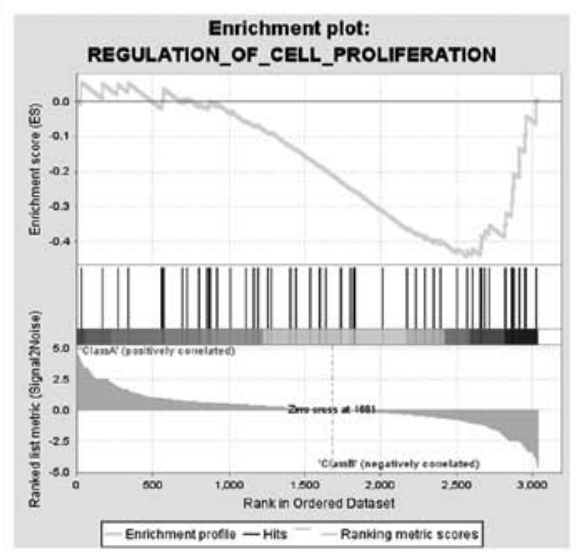

Figure 3. YY1 depletion represses PCa cell viability and colony forming in a miR-146a-assisted manner. (A-C) Western blot assays were performed to demonstrate the expression effect of siYY1, pCMV6 and sh-YY1 on PCa cell lines. GAPDH was used as endogenous control. (D and E) miR-146a expression by qRT-PCR verified the inhibitory efficiency of si-YY1+antiNC/si-YY1+anti-miR-146a in both cell lines. The cell lines were transfected with si-YY1/si-NC, pCMV6-YY1/pCMV6, and si-YY1+anti-NC/si-YY1+anti-miR-146a. Cell viability and colony forming were measured by MTT (F-K) and colony formation assays (L-N). (O) GSEA showed enriched expression of gene sets involved in cell proliferation in YY1 knocked-down cells. ${ }^{*} \mathrm{P}<0.05$.

signal in YY1 knocked down cells (Fig. 4E). KRAS is the downstream molecule of EFGR signaling pathway, which has been reported be the target of miR-146a in PCa (5). The negative correlation of KRAS and YY1 knockdown suggest the 

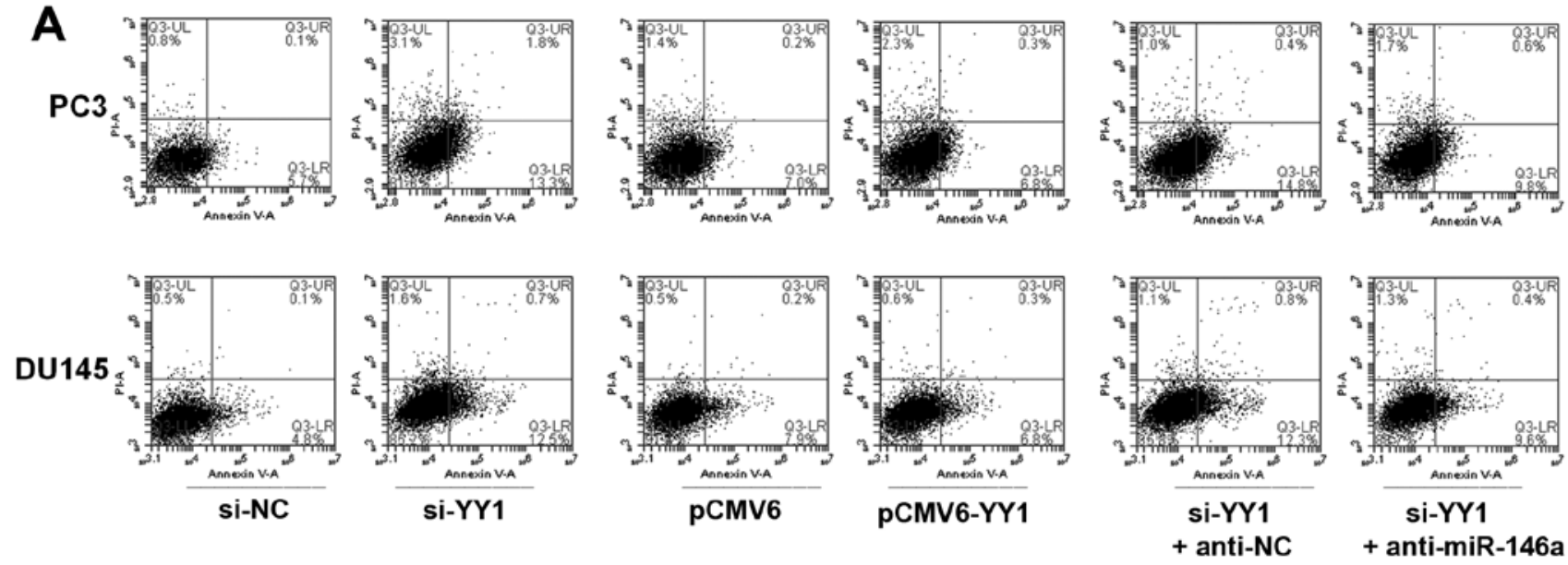

B

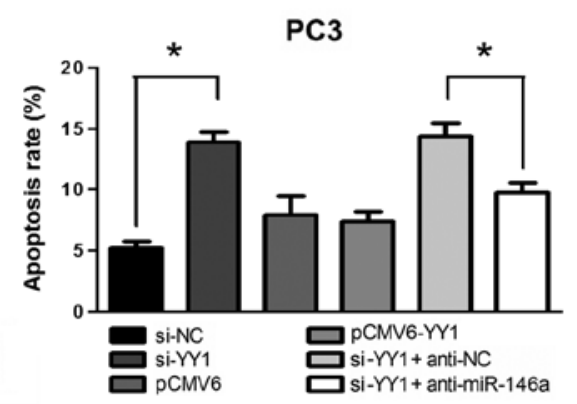

D

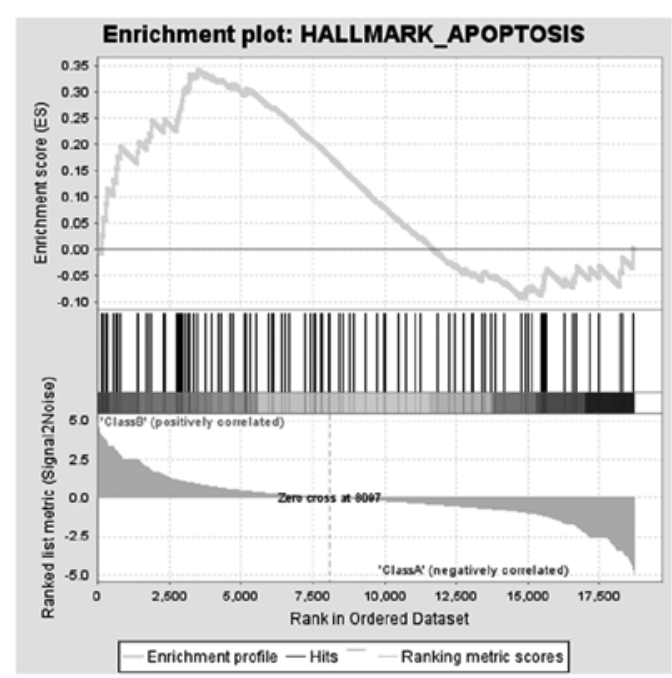

C
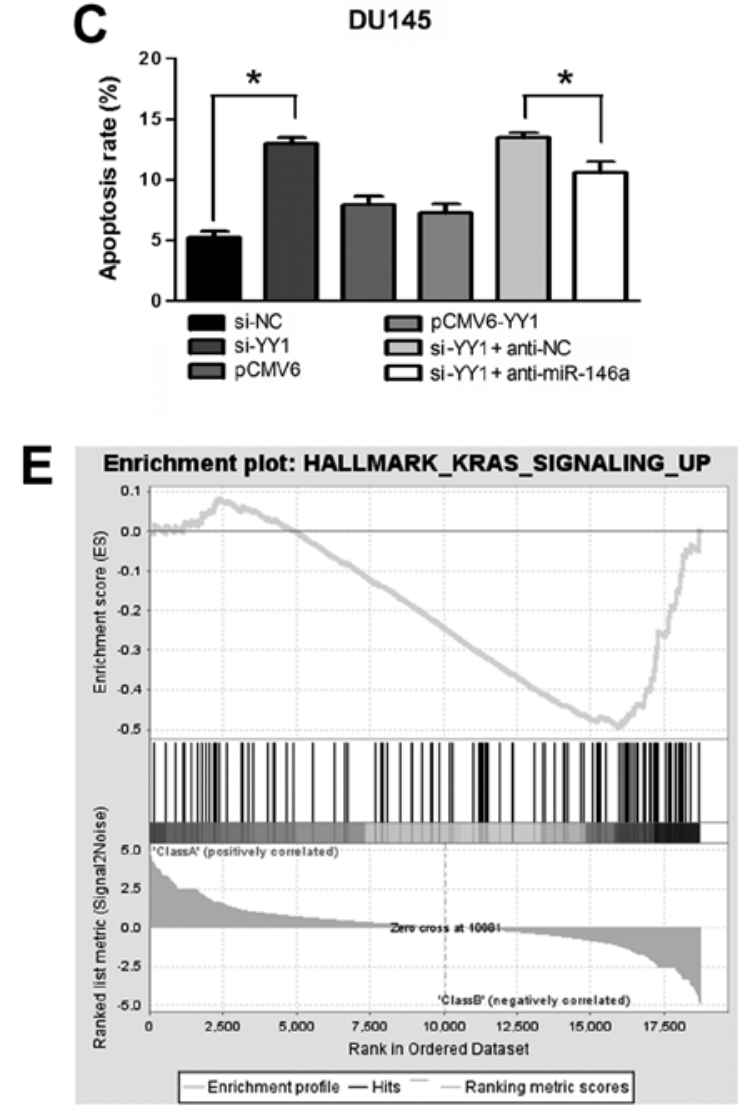

Figure 4. Depletion of YY1 induces cell apoptosis in a miR-146a-assisted manner. Both cell lines were transfected with si-YY1/si-NC, pCMV6-YY1/pCMV6, and si-YY1+anti-NC/si-YY1+anti-miR-146a. (A-C) Quantification of apoptosis with a fluorescence-activated cell sorter. Significance was determined by Student's t-test. "P<0.05. (D and E) GSEA showed enriched expression of gene sets involved in hallmarks of apoptosis and KRAS cell signals in YY1 knocked down cells.

potential regulation between YY1 and miR-146a. Moreover, miR-146a induced cell apoptosis in PCa cells by activating cleaved caspase-3 (15). Collectively, these results indicated that YY1 depletion induced cell apoptosis of PCa at least in a miR-146a-assisted manner.

Knockdown of YY1 suppresses xenografts growth accompanied by miR-146 upregulation. A stabilized sh-YY1-PC3 cell line was established, and the PCa cells were injected subcutaneously to either flank of the same nude mice. Tumor volumes were measured every week. Consequently, sh-YY1 inhibited tumor formation by volume starting from day 14 $(\mathrm{P}<0.05)$ (Fig. 5A and B). qRT-PCR indicated the significant upregulation of miR-146a expression in sh-YY1 xenografts $(\mathrm{p}<0.05)$ (Fig. 5C).

EZH2 was recruited by $Y Y 1$ at the promoter region of miR-146a, thereby repressing the miR-146a expression by participating in the transcriptional repression. We measured the expression of miR-146a through qRT-PCR to elucidate 


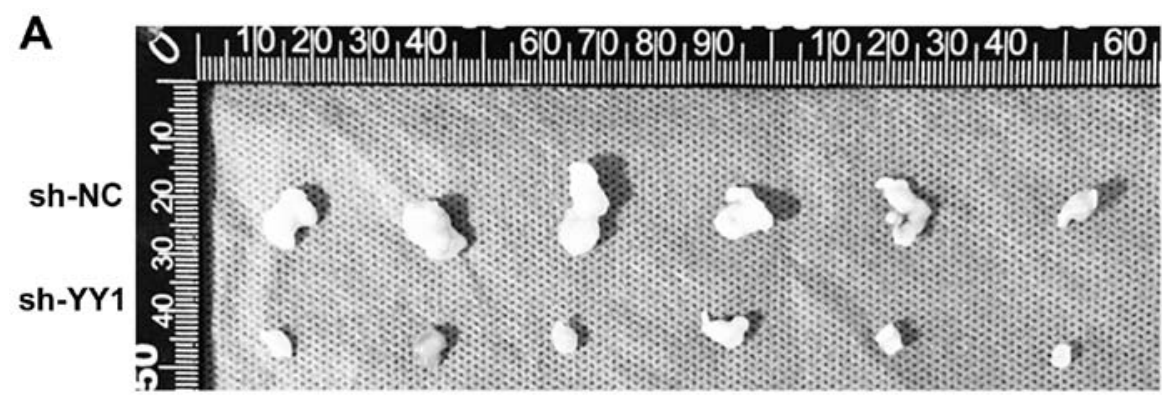

B
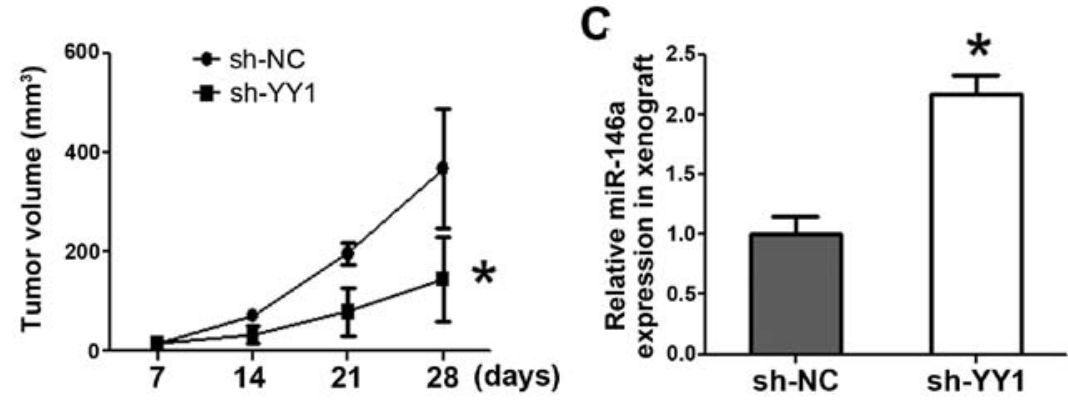

Figure 5. YY1 depletion suppresses xenografts growth accompanied by miR-146 upregulation. (A and B) After establishment of subcutaneous tumors, tumor volumes were measured every week. The tumor volume of the sh-YY1 group was significantly decreased compared with the sh-NC group. (C) qRT-PCR detected the increase of miR-146a expression in sh-YY1 xenografts. ${ }^{*} \mathrm{P}<0.05$.

the regulatory functions of YY1 and miR-146a further. Accordingly, miR-146a was significantly upregulated after si-YY1 treatment, whereas no significant change was observed after pCMV6-YY1 treatment (Fig. 6A-D). To elucidate the regulatory mechanism of miR-146a transcription process, the JARSPA database was consulted to investigate the concise regulatory mechanism of YY1-miR-146a (-2,000 bp upstream of miR-146a transcriptional start site, chr5:159895258, GRCH37). (Fig. 6E) One binding site of YY1 for the pre-miR146a promoter was predicted (profile score threshold, $80 \%$ ). A 1,262 bp fragment upstream of human pri-miR-146a was constructed and inserted into the luciferase reporter plasmid pGL3-basic-Vector. The plasmid was co-transfected with pCMV6 or pCMV6-YY1 into PC-3 cells. Luciferase reporter assay (Fig. 6F) showed that overexpression of YY1 led to a significant decrease in luciferase activity of the pGL3-miR146a-promoter plasmid in PC-3 cells. In addition, the ChIP assay (Fig. 6G) clearly showed YY1 combined at the miR-146a promoter region, thus further determined that YY1 could directly bind to the miR-146a promoter region (upstream), which indicated that YY1 could repress miR-146a expression through transcriptional suppression.

To further study the regulatory mechanism of miR-146a transcription, we performed GSEA to search for other co-repressors on the basis of YY1 depleted PC 3 cells. Notably, GSEA showed that the positively enriched expression of gene sets was repressed by EZH2 in YY1 depleted PC3 cells $(\mathrm{NES}=1.34, \mathrm{FDR}=0.02$ and $\mathrm{P}=0.02)$ (Fig. 7A), which suggested a potential co-relationship between YY1 and EZH2 during the transcriptional activity of PCa. EZH2 was reported to be positively co-operating with the tumorigenic transcription factor (i.e., YY1) (16). To further detect the influence of EZH2 on miR-146a expression, qRT-PCR was performed and demonstrated further upregulation of miR-146a after EZH2 knockdown on the basis of YY1 depletion, suggesting that
EZH2 participated in the YY1-mediated regulatory process (Fig. 7B). IP assays (Fig. 7C) were performed using nuclear protein from PC3 cells, and validated the directly combined effects of EZH2 and YY1 in the nucleus. Moreover, the IF provided a clear view of EZH2 and YY1 located within the cell structure. The general view by IF (Fig. 7D) of the YY1 and EZH2 conjunction inside the nucleus and cytoplasm was observed indicated that YY1 and EZH2 might jointly participate in some biological process. Together with the preceding ChIP and Luciferase results, we verified that EZH2 was recruited by YY1 at the promoter region of miR-146a and jointly repressed the miR-146a expression by participating in the transcriptional repression.

\section{Discussion}

miR-146a (miR-146a-5P) is a well-known anti-carcinoma cell signal modulator in prostate cancer (PCa) tissues. Our research group have investigated the mechanism of miR-146a in inhibiting PCa cells $(5,7,12)$. However, in this study, we focused on whether miR-146a expression can be regulated by pivotal transcriptional factors. By means of promoter analysis, YY1 was selected as the transcription factor with the most potential. We determined that YY1 was upregulated in PCa tissues with biochemical recurrence, high Gleason score, and low miR-146a expression. YY1 depletion inhibited cell viability and proliferation and induced cell apoptosis of PCa cells along with the upregulation of miR-146a. Through GSEA in YY1 knocked down PC3 cells, we observed the negatively enriched expression of gene sets, which were in conformity with the miR-146a function of PCa in cell proliferation (6), cell apoptosis (7), and KRAS signal (5) as previously reported.

As a transcription factor for miR-146a, YY1 was first reported by Seligson et al (17) to be overexpressed in PCa 
A

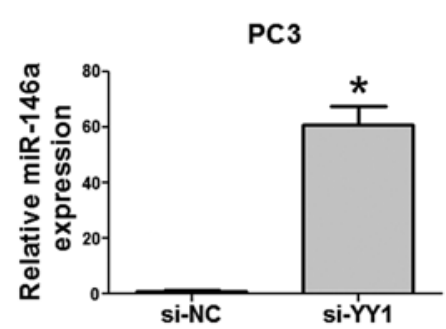

C

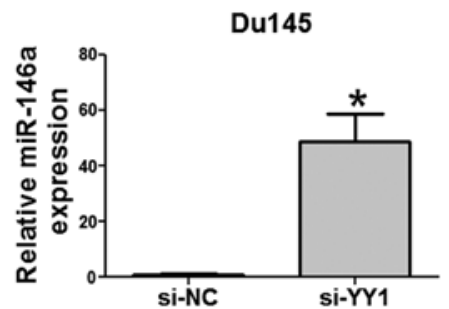

B

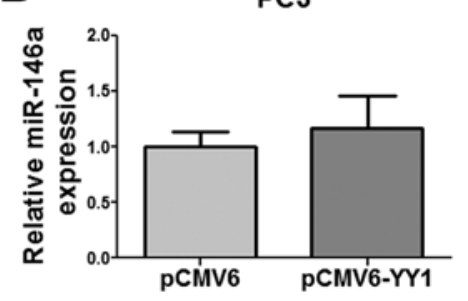

D

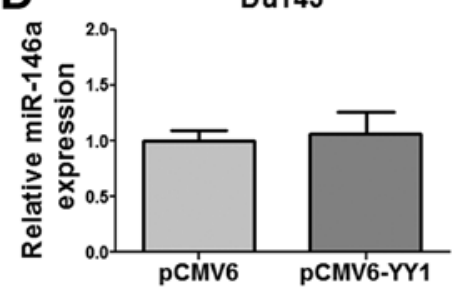

$E_{\text {pGL3-basic-miR-146a-promoter region (-1,262 bp) }}$

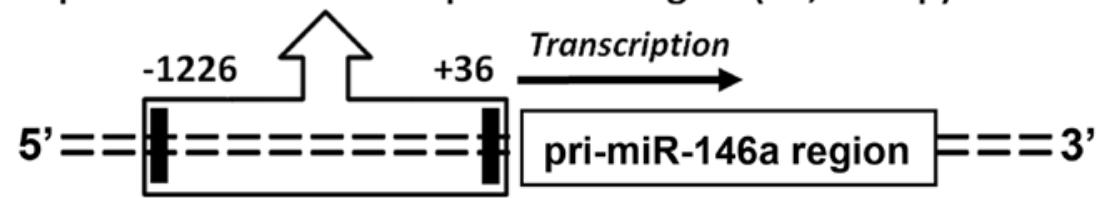

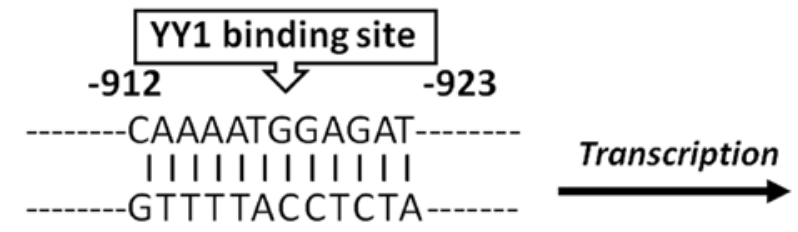

Wild-type

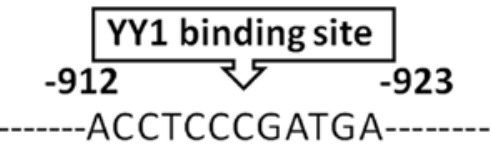

Mutant

| | | | | | | | | | | |

Transcription

F

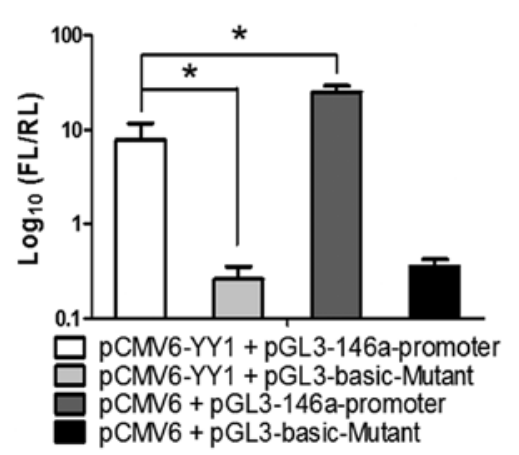

G

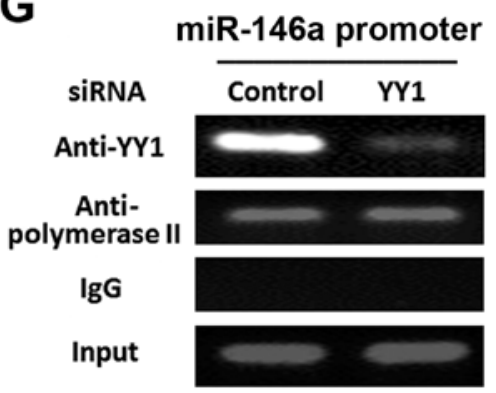

Figure 6. miR-146a is negatively regulated by YY1 at the transcriptional level. (A-D) miR-146a expression by qRT-PCR detected the effect of si-YY1/si-NC, pCMV6-YY1/pCMV6 in both PCa cell lines. (E) Schematic of putative YY1 binding motifs in the miR-146a promoter region (-1,262 bp upstream of the miR-146a promoter). Mutant binding motif for YY1 was chemically synthesized to generate the pGL3-basic-mutant reporter. (F) Luciferase reporter assay were performed to detect the junction of YY1 at the miR-146a promoter region (-912 to -923 bp before the transcription start site). pGL3-miR-146a reporter vector $(1 \mu \mathrm{g})$, together with $2 \mu \mathrm{g}$ of pCMV6-YY1 or pCMV6 were co-transfected into PC3 cells. Luciferase activities were determined and normalized to Renilla activity after transfection for $24 \mathrm{~h}$. ${ }^{*} \mathrm{P}<0.05$. (G) ChIP assays were used to assess YY1 binding site at the promoter region of miR-146a. The amounts of precipitated endogenous miR-146a promoter fragments were determined by PCR using the specific primer and visualized with gel electrophoresis.

cells compared with normal tissues. A certain level of YY1 inside the cell membrane is essential in maintaining basic biological functions, and YY1 also contributes to carcinogenesis (18). YY1 influences gene expression by directly or 
A

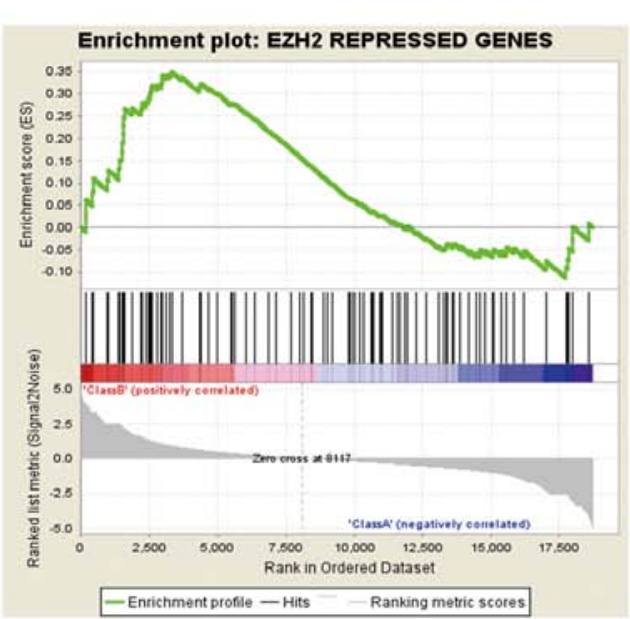

B

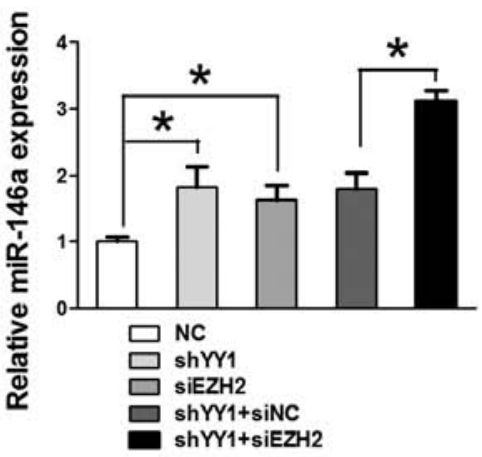

C

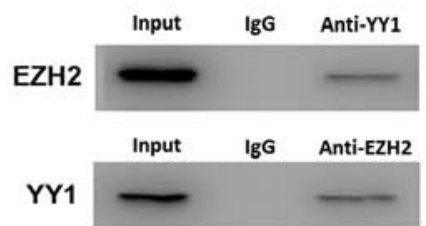

D

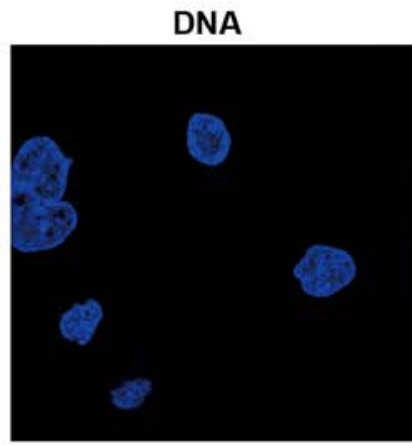

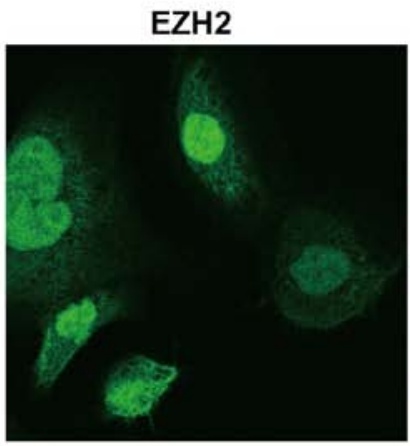
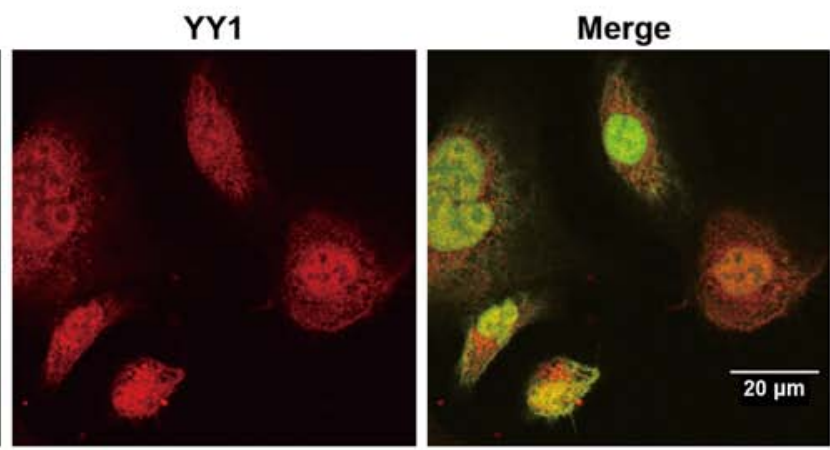

Figure 7. EZH2 is recruited at the promoter region of miR-146a by YY1, thereby repressing the expression of miR-146a. (A) Positively enriched expression of gene sets repressed by EZH2 are shown in YY1 knocked down PC3 cell line by GSEA. (B) Expression of miR-146a in sh-YY1 PC3 cells with EZH2 depletion by qRT-PCR showed further upregulation of miR-146a after EZH2 knockdown, suggested the potential role of EZH2 participating in the YY1-mediated regulatory process. "P<0.05. (C) IP were performed using nuclear extraction to detect the combination between EZH2 and YY1 in PC3 cells. (D) IF provide the general view of the YY1 and EZH2 inside the PC3 cells.

indirectly activating or inhibiting gene transcription (19) and activates a variety of oncogene factors by interacting with the transforming growth factor $\beta-3$ (20) and v-myc avian myelocytomatosis viral oncogene homolog (c-Myc) (21).

Notably, in this study, GSEA-represented gene sets suppressed by EZH2 regulation were positively enriched after YY1 depletion, indicating the potential relationship between essential transcriptional factors (i.e., EZH2 and YY1) and $\mathrm{PCa}$ cells. EZH2, a crucial component of polycomb repressor complex 2 (PRC2), is reported to be overexpressed in $\mathrm{PCa}$ cells (22). EZH2 represses gene expression by catalyzing the tri-methylation of histone H3 lysine 27 (H3K27me3) which regulates gene transcription epigenetically (23). The high expression level of EZH2 in clinically localized PCa tissues incur poor prognosis (24). Accumulating evidence indicates that PRC2 exerts strong oncogenic activity. These findings are proven by investigations on cell proliferation (25), cell invasion, maintenance of tumor-initiating cells (26), and tumor xenograft growth in vivo (27). Studies $(28,29)$ have shown that EZH2 participates in the pre-transcriptional process of tumor suppressor miRNAs (miR-181a, miR-203, and miR-200 families), thereby implying that a few dominant oncogenes (e.g., YY1) and pathways are involved in multiple cellular processes orchestrated through the regulatory network of EZH2.

As to the incapability of the overexpression of YY1 to enhance the carcinoma ability of PCa cells and to upregulate the expression of miR-146a, it was speculated to be caused by the resulting saturation effect on the YY1 regulatory system. Inside the cytoplasm, special miRNAs cannot be regulated by merely a single and simplistic regulator (e.g., YY1). For example, in the regulatory circuitry in renal cell carcinoma cells, the repression of CCAAT/enhancer-binding protein $\alpha$ $(\mathrm{C} / \mathrm{EBP} \alpha)$ induced by YY1 leads to a reduction in miR-34a expression; by contrast, miR-34a exerts a tumor-suppressive function by directly suppressing the oncogenic YY1, thereby forming a YY1-C/EBP $\alpha$-miR-34a positive feedback loop (30). Nevertheless, YY1 requires various co-transcriptional factors, such as histone deacetylase 4 (31) and EZH2 (16), to perform its function in the target genes epigenetic silencing process. As a result, overexpressed YY1 inside the cytoplasm alone has limited opportunity or access to the role of tumorigenic transcriptional factor, thereafter cannot enhance the YY1-associated transcriptional repression.

In PCa, miR-146a is reported to suppress cell viability via the nuclear factor $\kappa$-light-chain-enhancer of the activated B cells (NF- $\kappa B$ ) signaling pathway (8), whereas the classic NF- $\kappa B$ pathway activates the transcription of YY1 (32). Together with the preceding results, a dynamic YY1/EZH2miR-146a-NF- $\kappa$ B feedback is hypothesized to modulate the cell viability and proliferation of PCa cell lines. In addition, as the data shown in the assays, the miR-146a inhibitor cannot entirely rescue all changes in cell biological functions after 
YY1 depletion, suggesting there might be other existing target pathways affecting the PCa cell biological function.

Considering all of the results, we suggest that the recruitment of EZH2 by YY1 inhibits the miR-146a transcriptional activity in PCa cells, and YY1 depletion represses PCa cell viability and proliferation and induces apoptosis at least in a miR-146a-assisted manner. This result may provide a promising strategy for PCa treatment.

\section{Acknowledgements}

This work was supported by grants of National Natural Science Foundation of China (81202034 and 81572517) and the Scientific Research Innovation Project of University in Jiangsu Province (KYLX15_0184).

\section{References}

1. Siegel RL, Miller KD and Jemal A: Cancer statistics, 2015. CA Cancer J Clin 65: 5-29, 2015.

2. Katzenwadel A and Wolf P: Androgen deprivation of prostate cancer: Leading to a therapeutic dead end. Cancer Lett 367: 12-17, 2015.

3. Hua K, Jin J, Zhang $\mathrm{H}$, Zhao B, Wu C, Xu H and Fang L: MicroRNA-7 inhibits proliferation, migration and invasion of thyroid papillary cancer cells via targeting CKS2. Int J Oncol 49: 1531-1540, 2016

4. Li J, Yang X, Guan H, Mizokami A, Keller ET, Xu X, Liu X, Tan J, $\mathrm{Hu} \mathrm{L}, \mathrm{Lu} \mathrm{Y}$, et al: Exosome-derived microRNAs contribute to prostate cancer chemoresistance. Int J Oncol 49: 838-846, 2016.

5. Xu B, Wang N, Wang X, Tong N, Shao N, Tao J, Li P, Niu X, Feng N, Zhang L, et al: MiR-146a suppresses tumor growth and progression by targeting EGFR pathway and in a p-ERKdependent manner in castration-resistant prostate cancer. Prostate 72: 1171-1178, 2012.

6. Sun Q, Zhao X, Liu X, Wang Y, Huang J, Jiang B, Chen Q and $\mathrm{Yu} \mathrm{J}$ : miR-146a functions as a tumor suppressor in prostate cancer by targeting Rac1. Prostate 74: 1613-1621, 2014.

7. Xu B, Huang Y, Niu X, Tao T, Jiang L, Tong N, Chen S, Liu N, Zhu W and Chen M: Hsa-miR-146a-5p modulates androgenindependent prostate cancer cells apoptosis by targeting ROCK1. Prostate 75: 1896-1903, 2015.

8. Liu R, Yi B, Wei S, Yang WH, Hart KM, Chauhan P, Zhang W, Mao X, Liu X, Liu CG, et al: FOXP3-miR-146-NF-кB axis and therapy for precancerous lesions in prostate. Cancer Res 75 . 1714-1724, 2015

9. Bhalla SS, Robitaille L and Nemer M: Cooperative activation by GATA-4 and YY1 of the cardiac B-type natriuretic peptide promoter. J Biol Chem 276: 11439-11445, 2001.

10. Lee MY, Lu A and Gudas LJ: Transcriptional regulation of Rex1 (zfp42) in normal prostate epithelial cells and prostate cancer cells. J Cell Physiol 224: 17-27, 2010.

11. Luo J, Zhou X, Ge X, Liu P, Cao J, Lu X, Ling Y and Zhang S: Upregulation of Ying Yang 1 (YY1) suppresses esophageal squamous cell carcinoma development through heme oxygenase-1. Cancer Sci 104: 1544-1551, 2013.

12. Xu B, Feng NH, Li PC, Tao J, Wu D, Zhang ZD, Tong N, Wang JF, Song NH, Zhang W, et al: A functional polymorphism in Pre-miR-146a gene is associated with prostate cancer risk and mature miR-146a expression in vivo. Prostate 70: 467-472, 2010.

13. Tao T, Wang Y, Luo H, Yao L, Wang L, Wang J, Yan W, Zhang J, Wang H, Shi Y, et al: Involvement of FOS-mediated $\mathrm{miR}-181 \mathrm{~b} / \mathrm{miR}-21$ signalling in the progression of malignant gliomas. Eur J Cancer 49: 3055-3063, 2013.
14. Taganov KD, Boldin MP, Chang KJ and Baltimore D: NF-kappaB-dependent induction of microRNA miR-146, an inhibitor targeted to signaling proteins of innate immune responses. Proc Natl Acad Sci USA 103: 12481-12486, 2006.

15. Zhang B, Wang LL, Ren RJ, Dammer EB, Zhang YF, Huang Y, Chen SD and Wang G: MicroRNA-146a represses LRP2 translation and leads to cell apoptosis in Alzheimer's disease. FEBS Lett 590: 2190-2200, 2016.

16. Tsang DP, Wu WK, Kang W, Lee YY, Wu F, Yu Z, Xiong L, Chan AW, Tong JH, Yang W, et al: Yin Yang 1-mediated epigenetic silencing of tumour-suppressive microRNAs activates nuclear factor- $\mathrm{\kappa B}$ in hepatocellular carcinoma. J Pathol 238: 651-664, 2016.

17. Seligson D, Horvath S, Huerta-Yepez S, Hanna S, Garban H, Roberts A, Shi T, Liu X, Chia D, Goodglick L, et al: Expression of transcription factor Yin Yang 1 in prostate cancer. Int J Oncol 27: 131-141, 2005.

18. Deng Z, Cao P, Wan MM and Sui G: Yin Yang 1: A multifaceted protein beyond a transcription factor. Transcription 1: 81-84, 2010.

19. Thorvaldsen JL, Weaver JR and Bartolomei MS: A YY1 bridge for X inactivation. Cell 146: 11-13, 2011.

20. Caggia S, Libra M, Malaponte G and Cardile V: Modulation of YY1 and p53 expression by transforming growth factor- $\beta 3$ in prostate cell lines. Cytokine 56: 403-410, 2011.

21. Sankar N, Baluchamy S, Kadeppagari RK, Singhal G, Weitzman S and Thimmapaya B: p300 provides a corepressor function by cooperating with YY1 and HDAC3 to repress c-Myc. Oncogene 27: 5717-5728, 2008.

22. Aldiri I and Vetter ML: PRC2 during vertebrate organogenesis: A complex in transition. Dev Biol 367: 91-99, 2012.

23. Xiong X, Zhang J, Liang W, Cao W, Qin S, Dai L, Ye D and Liu Z: Fuse-binding protein 1 is a target of the EZH2 inhibitor GSK343, in osteosarcoma cells. Int J Oncol 49: 623-628, 2016.

24. Varambally S, Dhanasekaran SM, Zhou M, Barrette TR, Kumar-Sinha C, Sanda MG, Ghosh D, Pienta KJ, Sewalt RG, Otte AP, et al: The polycomb group protein EZH2 is involved in progression of prostate cancer. Nature 419: 624-629, 2002.

25. Bracken AP, Pasini D, Capra M, Prosperini E, Colli E and Helin K: EZH2 is downstream of the pRB-E2F pathway, essential for proliferation and amplified in cancer. EMBO J 22: 5323-5335, 2003.

26. Wen S, Tian J, Niu Y, Li L, Yeh S and Chang C: ASC-J9(®), and not Casodex or Enzalutamide, suppresses prostate cancer stem/progenitor cell invasion via altering the EZH2-STAT3 signals. Cancer Lett 376: 377-386, 2016.

27. Kleer CG, Cao Q, Varambally S, Shen R, Ota I, Tomlins SA, Ghosh D, Sewalt RG, Otte AP, Hayes DF, et al: EZH2 is a marker of aggressive breast cancer and promotes neoplastic transformation of breast epithelial cells. Proc Natl Acad Sci USA 100: 11606-11611, 2003.

28. Cao Q, Mani RS, Ateeq B, Dhanasekaran SM, Asangani IA, Prensner JR, Kim JH, Brenner JC, Jing X, Cao X, et al: Coordinated regulation of polycomb group complexes through microRNAs in cancer. Cancer Cell 20: 187-199, 2011.

29. Zhou L, Wang L, Lu L, Jiang P, Sun H and Wang H: A novel target of microRNA-29, Ring1 and YY1-binding protein (Rybp), negatively regulates skeletal myogenesis. J Biol Chem 287: 25255-25265, 2012.

30. Weng W, Wang M, Xie S, Long Y, Li F, Sun F, Yu Y and Li Z: YY1-C/EBP $\alpha$-miR34a regulatory circuitry is involved in renal cell carcinoma progression. Oncol Rep 31: 1921-1927, 2014.

31. Ren G, Zhang G, Dong Z, Liu Z, Li L, Feng Y, Su D, Zhang Y, Huang B and Lu J: Recruitment of HDAC4 by transcription factor YY1 represses HOXB13 to affect cell growth in AR-negative prostate cancers. Int J Biochem Cell Biol 41: 1094-1101, 2009.

32. Wang H, Garzon R, Sun H, Ladner KJ, Singh R, Dahlman J, Cheng A, Hall BM, Qualman SJ, Chandler DS, et al: NF-kappaBYY1-miR-29 regulatory circuitry in skeletal myogenesis and rhabdomyosarcoma. Cancer Cell 14: 369-381, 2008. 